\title{
Variation in timing of decisions to withdraw life- sustaining treatment in adult ICU patients from three centres in different geographies: Do clinical factors explain the difference?
}

\author{
W H Seligman, ${ }^{1}$ BA Hons, Oxon BM BCh, MRCP, MPH; OrcID 0000-0001-6061-7342; N Sadovnikoff, ${ }^{2}$ MD; OrcID 0000-0002-3887-2272; \\ I A Joubert, ${ }^{3} \mathrm{MB}$ BCh, DA(SA), FCA(SA) (Critical Care); P Hutton, ${ }^{4}$ BSc Hons, PG Cert, PG Diploma; \\ M Flint, ${ }^{3}$ BSc, HSc, MSc, PhD; OrcID 0000-0002-3005-5825; A M Courtwright, ${ }^{5}$ MD, PhD; OrcID 0000-0001-9511-5975; \\ K B Krishnamurthy, ${ }^{6} \mathrm{MD}$; A M Joseph, ${ }^{7} \mathrm{MD}$ MPH; OrcID 0000-0003-2768-0889; \\ S McKechnie, ${ }^{4} \mathrm{MB}$ ChB, BSc Hons, FRCA, PhD, DICM, FFICM \\ ${ }^{1}$ Imperial School of Anaesthesia, London, UK \\ ${ }^{2}$ Department of Anesthesiology, Perioperative and Pain Medicine, Brigham and Women's Hospital, Boston, Massachusetts, USA \\ ${ }^{3}$ Department of Anaesthesia and Perioperative Medicine, Division of Critical Care, University of Cape Town and Groote Schuur Hospital, South Africa \\ ${ }^{4}$ Adult Intensive Care Unit, Oxford University Hospitals NHS Foundation Trust, Oxford, UK \\ ${ }^{5}$ Division of Pulmonary and Critical Care Medicine, Hospital of the University of Pennsylvania, Philadelphia, USA \\ ${ }^{6}$ Department of Neurology, Beth Israel Deaconess Medical Center, Boston, Massachusetts, USA \\ ${ }^{7}$ Department of Critical Care Medicine, UPMC Children's Hospital of Pittsburgh, Pennsylvania, USA
}

Corresponding author: W H Seligman (seligmanw@gmail.com)

\begin{abstract}
Background. Decisions to withdraw life-sustaining treatment (WLST) are common in intensive care units (ICUs). Clinical and non-clinical factors are important, although the extent to which each plays a part is uncertain.

Objectives. To determine whether the timing of decisions to WLST varies between ICUs in a single centre in three countries and whether differences in timing are explained by differences in clinical decision-making.

Methods. The study involved a convenience sample of three adult ICUs - one in each of the UK, USA and South Africa (SA). Data were prospectively collected on patients whose life-sustaining treatment was withdrawn over three months. The timing of decisions was collected, as were patients' premorbid functional status and illness severity 24 hours prior to decision to WLST. Multivariate analysis was used to identify factors associated with decisions to WLST. Clinicians participated in interviews involving hypothetical case studies devoid of non-clinical factors. Results. Deaths following WLST accounted for $23 \%$ of all deaths during the study period at the USA site v. 37\% (UK site) and $70 \%$ (SA site) ( $p<0.0010$ across the three sites). Length of stay (LOS) prior to WLST decision varied between sites. Controlling for performance status, age, and illness severity, study site predicted LOS prior to decision $(p<0.0010)$. In the hypothetical cases, LOS prior to WLST was higher for USA clinicians $(p<0.017)$.

Conclusion. There is variation in the proportion of ICU patients in whom WLST occurs and the timing of these decisions between sites; differences in clinical decision-making may explain the variation observed, although clinical and non-clinical factors are inextricably linked.

Keywords: intensive care, ethics, withdrawal of life-sustaining treatment.
\end{abstract}

South Afr J Crit Care 2020;36(1):18-22. https://doi.org/10.7196/SAJCC.2020.v36i1.393

\section{Contribution of study}

This study has identified variation in the timing of decisions to withdraw life-sustaining treatment in adult ICUs in three centres in three different healthcare systems. Although differences in clinical decision-making likely explain some of the variation, non-clinical factors (relating to the society in which the clinicians live and work) may also play a part.

Decisions to withdraw life-sustaining treatment (WLST) are common in intensive care and precede the majority of deaths in European intensive care units (ICUs) ${ }^{[1]}$ Deciding when to shift the goals of therapy away from sustaining life towards end-of-life care may be more complex than deciding whom to admit to the ICU in the first place. ${ }^{[2]}$ The timing of the decision to WLST is likely to be influenced by a number of factors, some of which are directly related to the patient, which in this study we refer to as 'clinical factors', and some of which concern the department, hospital, geography and society within which the patient is being treated, which we refer to as 'non-clinical factors.'

There is evidence that clinicians' practice is influenced by non-clinical factors. For example, a survey of 862 USA doctors found that physicians were more willing to WLST if the clinicians were young, practised in a tertiary care setting or spent a greater proportion of their time in 
clinical practice compared with in research or administration. ${ }^{[3]}$ Where one practises may also be a contributing factor. In a questionnaire assessing clinicians' response to hypothetical clinical situations, Japanese physicians practising in the USA responded more similarly to American physicians trained in the USA than Japanese physicians in Japan. ${ }^{[4]}$ It is clear, therefore, that decisions to WLST are affected both by clinical and non-clinical factors. Given the complexity of the WLST decisionmaking process and the number of possible factors that are involved in these decisions, it is unsurprising that there exists significant variability in end-of-life practices between countries. ${ }^{[1,5]}$ Studying the ICU length of stay (LOS) prior to decision to WLST in different units may help us to identify important reasons behind the variability seen in these international comparisons.

\section{Objectives}

To determine the proportion of patients admitted to ICU in whom a decision to WLST was made in each of the study sites over a threemonth period, the timing of decisions to WLST following admission to ICU in each of the study sites and whether any difference in the timing of decisions to WLST following admission to ICU can be explained by clinical factors.

\section{Methods \\ Study locations and context}

We selected three similar institutions (all tertiary referral centres receiving acute medical, surgical and trauma admissions as well as elective surgical patients) in geographically, financially and culturally distinct countries. The sites represent a convenience sample. John Radcliffe Hospital (JRH), in the UK, is a mixed adult medical-surgical unit in Oxford affiliated with the University of Oxford. Brigham and Women's Hospital (BWH), in the USA, is a mixed adult medical-surgical unit in Boston affiliated with Harvard University. Groote Schuur Hospital (GSH), in South Africa (SA), is a mixed adult medical-surgical unit in Cape Town affiliated with the University of Cape Town (UCT).

The study was approved by the University of Oxford Medical Sciences Division Research Ethics Committee (ref. no. MSDIDREC-C1-2014-201) in the UK, Human Research Ethics Committee, UCT (ref. no. 197/2015) in SA and Partners Human Research Committee (ref. no. 2015P001959/BWH) in the USA.

\section{Prospective observational study}

Over the course of a three-month period in each study centre, data were captured prospectively in order to identify:

- the proportion of patients whose life-sustaining treatment was withdrawn (number of patients who died during the study period following a decision to WLST as a proportion of the total number of patients who died during the study period)

- the median LOS on ICU prior to the decision to WLST being made

- demographics, including age, pre-admission WHO performance status and illness severity (sequential organ failure assessment (SOFA) score) for the 24-hour period prior to the decision being made, of the patients whose treatment was withdrawn in each ICU. SOFA was chosen as the indicator of illness severity in this study due to its simplicity and ease of standardised recording.

Each day the study was running, a form was completed immediately after the daily ward round indicating whether any decisions had been made regarding treatment withdrawal. Any patient in whom a decision to WLST was made during the study period was included apart from patients meeting criteria for brainstem death. A decision to WLST was recorded if any of the following decisions were made:

- removal of ventilatory support with palliative intent (either extubation or cessation of non-invasive ventilator support)

- discontinuation of vasoactive drugs with palliative intent

- discontinuation of renal replacement therapy with palliative intent or

- discontinuation of life-sustaining non-palliative medications with palliative intent.

For patients in whom a decision to WLST was made, the patient's age, gender, admission diagnosis, LOS on ICU before the decision, preadmission WHO SOFA scores were recorded.

\section{Interviews with ICU physicians}

To identify the relative importance of clinical factors, the chief investigator or local principal investigator conducted structured interviews with senior ICU physicians (attending physicians/consultants). Ten senior ICU clinicians from the USA, seven from the UK and six from the SA sites agreed to be interviewed. In these interviews, three identical hypothetical case studies were presented. The case studies were devoid of external pressures (for e.g., the clinicians were told that there were unlimited ICU beds and that the patients had no family) to determine whether in an environment free from external pressures, ICU physicians make similar clinical decisions irrespective of where they practise. The case studies were developed by the chief investigator and UK principal investigator incorporating feedback from peer review. The case studies were designed to include two common medical admission diagnoses, namely sepsis from lower respiratory tract infection (case study 1 ) and out-of-hospital cardiac arrest (case study 2), and one common surgical admission diagnosis, perforated diverticular disease (case study 3 ). Pre-admission WHO performance status was the same across the case studies.

The interviews were structured, and the interviewer presented clinical data on admission, and on subsequent days. The clinician was asked to verbalise at which point he/she would first contemplate WLST. The interviews were conducted on the telephone or in person and responses recorded using pseudonyms.

\section{Statistical analysis}

All statistical analyses were performed using STATA 15 (StataCorp., USA). Kruskal-Wallis $\mathrm{H}$ tests (KWT) were used to compare the demographics of patients included at the three study sites, including age, gender, WHO performance status, SOFA score and LOS. Fisher's exact test was used to compare the units in terms of the percentage of patients who died during study period following WLST as a proportion of total deaths during the study period. Wilcoxon rank sum tests were used on data generated from interviews with clinicians to perform pairwise comparisons between sites to determine whether there was a difference in the time point at which they would WLST in the hypothetical clinical cases. Multivariate linear regression analysis with study site as a dummy variable (USA chosen as reference population) was used to determine whether study site was a significant explanatory variable for LOS on ICU prior to WLST in our samples. Other covariates included in the analysis were: pre-admission WHO performance status, age and SOFA score in the 24-hour period prior to the decision to withdraw. LOS data underwent logarithmic transformation before being included in the linear regression since the data were not normally distributed. A $p<0.05$ represented statistical significance in all tests except for when 
performing multiple pairwise comparisons, in which case a Bonferroni correction was applied and the relevant statistically significant $p$-value is indicated.

\section{Results}

During the three-month study periods, there were 823 admissions to ICU in the USA site (maximum of 35 beds), 209 admissions in the UK site (maximum of 16 beds) and 107 admissions in the SA site (maximum of 19 beds). There was a statistically significant difference $(p<0.001)$ between the proportions of deaths following WLST as a function of total deaths during the study period between the three sites (Table 1).

Median LOS prior to the decision to WLST was significantly longer in the USA site than in the UK or SA sites even when controlling for illness severity, age, and pre-admission WHO performance status $(p<0.001)$. This is notwithstanding the significant differences between units in median age and median SOFA score 24 hours prior to decision to WLST.

In two of the three hypothetical clinical vignettes, fewer SA physicians interviewed would have admitted the patient to ICU compared with the UK and USA physicians interviewed. Of those patients whom the physicians would admit, in each of the three cases, the physicians in the
USA began contemplating WLST significantly later than the physicians interviewed from the other sites (Table 2). There was a significant difference in median LOS between the UK and USA clinicians for all three cases $(p<0.017$ for each) and between the USA and SA clinicians in case study 3 ( $p=0.015)$. There was no difference between the UK and SA clinicians in case studies 1 or 3 (no statistical analysis was performed on the SA cohort for case study 2 due to the small sample size resulting from the few respondents who would have admitted the patient to ICU).

\section{Discussion}

In this small prospective international multicentre study of decisions to WLST in adult ICUs, there were proportionally more decisions to WLST in the SA site than in the USA or UK sites. Withdrawal decisions were made following a significantly longer ICU LOS in the USA site compared with the UK or SA, even following correction for pre-admission WHO performance status and illness severity. This remained the case even after adding age as an additional co-variate in the regression model despite the small sample size.

When presented with hypothetical clinical case studies devoid, at least in theory, of non-clinical considerations, clinicians at the USA site stated they would first consider WLST significantly later than clinicians at the

Table 1. Characteristics of the study patients, during the study period, in whom life-sustaining treatment was withdrawn

\begin{tabular}{|c|c|c|c|c|}
\hline & BWH (USA) & JRH (UK) & GSH (SA) & $\mathrm{KWT}^{*}$ \\
\hline ICU beds per unit $(n)$ & 35 & 16 & 19 & $\mathrm{n} / \mathrm{a}$ \\
\hline Admissions $(n)$ & 823 & 209 & 107 & $\mathrm{n} / \mathrm{a}$ \\
\hline Deaths, $n(\%)$ of all patients admitted to ICU & $107(13)$ & $27(13)$ & $20(19)$ & $p=0.27$ \\
\hline Deaths following WLST, $n(\%)$ of all ICU deaths & $25(23)$ & $10(37)$ & $14(70)$ & $p<0.001$ \\
\hline \multicolumn{5}{|l|}{ Breakdown of patients who had WLST by ICU admission diagnosis } \\
\hline Respiratory failure & 28 & 20 & 21 & \multirow{8}{*}{$p=0.006$} \\
\hline Trauma & 4 & 10 & 43 & \\
\hline Sepsis/multi-organ failure & 28 & 20 & 21 & \\
\hline Cardiac arrest & 24 & 20 & 0 & \\
\hline Liver failure & 8 & 0 & 0.0 & \\
\hline Renal failure & 0 & 0 & 14 & \\
\hline Poisoning & 8 & 0 & 0 & \\
\hline Emergency surgery & 0 & 30 & 0 & \\
\hline Age (years), median (IQR) & $74(70-81)$ & $67(62-76)$ & $33(30-44)$ & $p<0.0001$ \\
\hline \multicolumn{5}{|l|}{ Gender } \\
\hline Male & 10 & 7 & 10 & \multirow{2}{*}{$p=0.9$} \\
\hline Female & 15 & 3 & 4 & \\
\hline Median pre-admission WHO performance status, $n$ (IQR) & $1(1-2)$ & $1(1-2)$ & $2(1-3)$ & $p=0.7$ \\
\hline Median SOFA score 24 hours prior to decision to withdraw, $n$ (IQR) & $8(6-11)$ & $13(8-16)$ & $12(11-15)$ & $p=0.006$ \\
\hline Median LOS (days) prior to decision to withdraw, $n$ (IQR) & $8(6-11)$ & $3(2-4)$ & $3(2-4)$ & $p=0.0001$ \\
\hline
\end{tabular}

Table 2. Differences in hypothetical decision to admit to ICU and timing of decision to WLST between study sites

\begin{tabular}{|c|c|c|c|c|c|c|}
\hline \multirow[b]{2}{*}{ Case study ${ }^{*}$} & \multicolumn{3}{|c|}{ Clinicians who would admit the patient to ICU, $n(\%)^{\dagger}$} & \multicolumn{3}{|c|}{ Number of days in ICU prior to decision to WLST, median (IQR) } \\
\hline & USA $(n=10)$ & UK $(n=7)$ & SA $(n=6)$ & USA & UK & SA \\
\hline 1 & $10(100)$ & $7(100)$ & $3(50)^{\ddagger}$ & $8(6-9)$ & $4(3-5)^{5}$ & $4(3-5)$ \\
\hline 2 & $10(100)$ & $7(100)$ & $1(17)^{\ddagger}$ & $5(4-7)$ & $2(1-3)^{5}$ & $3(3-3)$ \\
\hline 3 & $10(100)$ & $7(100)$ & $14(83)$ & $6(4-9)$ & $4(4-5)^{5}$ & $3(2-4)^{5}$ \\
\hline \multicolumn{7}{|c|}{$\begin{array}{l}\text { ICU = intensive care unit; WLST = withdrawal of life-sustaining treatment; } \mathrm{SA}=\text { South Africa. } \\
{ }^{*} \text { Case study } 1 \text { : sepsis from lower respiratory tract infection; Case study 2: out-of-hospital cardiac arrest; Case study 3: perforated diverticular disease. } \\
\text { †Statistical significance was defined as } p<0.0167 \text {, for both comparisons, due to Bonferroni correction for multiple pairwise comparisons. } \\
\text { "Represents statistical significance v. USA as reference population (for \% of clinicians who would admit the patient to ICU). } \\
\text { } \text { Represents statistical significance v. USA as reference population (for median length of stay prior to decision to WLST). }\end{array}$} \\
\hline
\end{tabular}


UK and SA sites. This could suggest that differences in clinical decisionmaking explain the observed increased length of ICU stay prior to WLST in the USA site.

However, if the observed differences in LOS before decision to WLST are the result of differences in clinical decision-making alone, it is difficult to explain why the SA cohort with patients who were younger and had less physiological derangement than, for example the UK cohort, had a higher proportion of WLST decisions. It is worth noting that as data collection took place over the festive period in SA, there was a significant over-representation of trauma-related ICU admissions in the patients whose life-sustaining treatment was withdrawn. This likely explains the significantly younger cohort of patients compared with the other sites. Further, although the peak SOFA score for SA patients may have occurred earlier in the admission and, therefore, might not have been captured, it is also possible that our observation reflects the challenging societal, cultural and political climate in SA and the inextricable link between clinical and non-clinical factors. This hypothesis is supported by the much higher proportion of all deaths during the study period preceded by WLST in the SA centre compared with the other centres.

There are significant cultural, societal, religious and economic differences between the three sites studied. SA is a culturally and economically diverse country suffering from the epidemics of HIV/ AIDS and trauma. Most of the population is served by the staterun public health system, which is resource-constrained and has proportionally few ICU beds. ${ }^{[6]}$ The prospect of regaining functional independence is thought to be a particularly important outcome in $\mathrm{SA}$, as long-term care facilities are minimal and there is limited longterm government support. ${ }^{[7]}$ Distributive justice takes on particular importance in this highly resource-constrained setting. ${ }^{[8]}$ There are 25 ICU beds per 100000 people in the USA v. 5 ICU beds per 100000 in the UK and 2 public ICU beds per 100000 people in SA. ${ }^{[9]}$ Spending on intensive care makes up nearly $1 \%$ of GDP in the USA, ${ }^{[10]}$ whereas it accounts for less than $0.1 \%$ of GDP in the UK. ${ }^{[11]}$

The significant differences in availability of ICU beds across countries may explain our finding from the hypothetical case interviews that admission decisions vary between countries. For instance, fewer SA respondents to our case studies would have admitted these hypothetical patients to the ICU in the first instance. Although our study did not seek to investigate differences in admission practice between centres in different countries, this is an area of possible future work.

The relative dearth of ICU beds in the UK and SA compared with the USA also likely explains previous findings showing that patients admitted to ICUs in the UK and SA have more physiologic derangements than in the USA. ${ }^{[12]}$ We might expect doctors in healthcare systems with fewer ICU beds to make proportionately more decisions to WLST. Indeed, in our study, the SA site made proportionally more withdrawal decisions than did the other sites. The USA site made fewer withdrawal decisions than did the UK site, consistent with having a greater number of available ICU beds and with having lower illness severity 24 hours prior to decision to WLST. Perhaps the relatively low SOFA scores 24 hours prior to withdrawal in the USA population reflects these decisions being made later on during a patient's ICU admission, when severity of illness may be less than earlier on in a patient's admission, which is when withdrawals occurred in the UK and SA sites. Additionally, perhaps because there are proportionately more critical care beds in the USA, the threshold for being admitted to ICU is lower and, therefore, patients' illness severity scores even at time of WLST are lower than in the other centres. This assertion is supported by a large retrospective cohort study of 172785 ICU admissions showing that patients admitted to ICUs in the USA have a significantly lower illness severity score than patients admitted to UK ICUs. ${ }^{[13]}$

Our finding that decisions to WLST are made at different times during patients' ICU stay in different countries, e.g. between USA and $\mathrm{UK}$, is in accordance with previously published data. In the ETHICUS study, decisions to limit life-sustaining therapy were made several days later in southern European countries than in northern or central European countries. ${ }^{[1]}$ The authors put forward several reasons as to why this might be the case, including different religions and cultures, different physician values and practices, difference in case mix, and ongoing evolution of end-of-life practices occurring at different rates and to different degrees among various countries. ${ }^{[1]}$ It is surprising that in our study, unlike in a previous study, ${ }^{[14]}$ there was no significant difference between the timing of decisions to withdraw life-sustaining treatment between the UK and SA centres, particularly given the great pressure on critical care resources in SA. While pressures do exist in the UK, they are nevertheless less intense than in SA, with a significantly lower density of ICU beds. Our finding of no difference in LOS prior to WLST in the UK and SA centres likely represents the small sample size of this study but may also suggest limited importance of 'non-clinical' factors in the timing of decisions. That is to say, if scarcity of resources was an important factor in the timing of decisions to WLST, one would expect there to be a difference between the LOS in the UK and SA, where resources are even scarcer.

There is, of course, a difficulty in separating 'non-clinical' from 'clinical' factors since physicians' practice patterns are likely heavily shaped by cultural factors, both specific to medicine and more broadly. A patient's level of 'illness' is therefore a matter of perception, driven in part by non-clinical factors. Consequently, despite the methodology used in this study explicitly attempting to discern the relative importance of clinical and non-clinical factors in explaining decision-making, it is not possible to disentangle the two issues fully.

Furthermore, there is likely to be variability around decisions to WLST even within the same ICU let alone across different ICUs. Our data provide some evidence for this. For instance, the time range in which US clinicians would first consider WLST in each of the hypothetical case studies was as great as five days. Significant intra-ICU variability may reduce the validity of inter-ICU comparisons.

\section{Study limitations}

The sites included in the study represent a convenience sample and were not selected at random. The sites are not representative of all hospitals in their associated countries and it is difficult to exclude selection bias. While the hospitals represent major academic centres in large cities in the USA, UK and SA, previous work has identified significant variations in practice within borders. ${ }^{[15]}$ In order to make stronger comparisons between the three countries in the study, it would be advantageous to include multiple sites in each country, representing not only academic centres but also rural/district hospitals. Furthermore, the investigators were not blinded to study site when collecting and analysing data. This is another potential source of bias.

The study is further limited by its small sample size and the short time period for data collection in each centre. Furthermore, as data were collected at different time points, it is not possible to exclude seasonal variation in ICU admissions as a possible confounding factor. Indeed, $43 \%$ of the patients who had WLST in the SA site were trauma patients. Ideally, future studies would collect data over a longer period of time and 
therefore have a larger sample size. It would also have been informative to collect data from all centres on admission illness severity scores as well as severity scores $24 \mathrm{hrs}$ prior to WLST.

In future, it may be helpful to present cases where a centre has decided to WLST to the other study centres to ascertain whether a decision to WLST would have been made at the same time or not across the different units. It may also be helpful to survey a large number of ICU clinicians working in different geographies about their perception of the influence of various clinical and non-clinical factors on the timing of decisions to WLST.

\section{Conclusion}

Decisions to WLST in adult ICUs are necessarily complex and are likely impacted by factors related to the patient being treated as well as to the person treating them and the healthcare system and society of which they are both a part. In this small study, we have demonstrated geographic variation in the proportion of patients whose life-sustaining treatment is withdrawn, and the timing of those decisions in two of the three centres. It is interesting that the LOS prior to WLST in the SA and UK sites was similar despite there being significant differences between the two countries. We have also identified differences in hypothetical clinical decision-making between clinicians at the study centres, which might help to explain the variation in practice.

Overall, while this was a non-blinded and non-randomised study and, therefore, should be used to generate hypotheses rather than test them, this is the first study that attempts to explore fully how differences in clinical factors may impact difficult WLST decisions. Further work should be carried out to investigate the influence of clinical and nonclinical factors on decision-making.

Declaration. The datasets used and/or analysed during the current study are available from the corresponding author on reasonable request. Acknowledgements. The authors would like to acknowledge Jodie Smythe and Elaine Armstrong for their help with data collection, and Rie Maurer for assistance with statistical analysis.
Author contributions. WHS and SM conceived of the study. WHS was the Chief Investigator. IJ, NS and SM acted as Local PIs in each of the study sites. AC, $\mathrm{PH}, \mathrm{MF}$ and $\mathrm{KBK}$ were responsible for the day-to-day running of the study in each site. AJ and WHS analysed the data and conducted statistical analysis. WHS wrote the first draft, which was subsequently reviewed by all authors.

Funding. WHS received funding from the Association of Anaesthetists of Great Britain and Ireland, Walter Guinness Trust and British Medical and Dental Students' Trust which was used to support travel related to the study. The funding bodies did not play any role in the design, conduct or analysis of the study.

Conflicts of interests. None.

1. Sprung CL, Cohen SL, Sjokvist P, et al. End-of-life practices in European intensive care units: The ETHICUS Study. JAMA 2003;290(6):790-797. https://doi.org/10.1001/jama.290.6.790

2. Wilkinson DJC, Savulescu J. Knowing when to stop: Futility in the intensive care unit. Curr Opin Anaesthesiol 2011;24(2):160-165. https://doi.org/10.1097\%2FACO.0b013e328343c5af

3. Christakis NA, Asch DA. Physician characteristics associated with decisions to withdraw life support. Am J Public Health 1995;85(3):367-371. https://doi.org/10.2105\%2Fajph.85.3.367

4. Asai A, Fukuhara S, Lo B. Attitudes of Japanese and Japanese-American physicians towards lifesustaining treatment. Lancet 1995;346(8971):356-359.

5. Cook DJ, Guyatt G, Rocker G, et al. Cardiopulmonary resuscitation directives on admission to intensive-care unit: An international observational study. Lancet 2001;358(9297):1941-1945. https:// doi.org/10.1016/s0140-6736(01)06960-4

6. Bhagwanjee S. Audit of Intensive Care utilisation in KwaZulu Natal: Focus on King Edward VIII and Addington Hospitals. Presented to KZN Healthcare Task Team 1998.

7. Morgan J. How do you decide when to withdraw life support? Lancet Respir Med 2015;3(6):430-431

8. McMillan J, Hope T. Justice-based obligations in intensive care. Lancet 2010;375(9721):1156-1157. https://doi.org/10.1016/s0140-6736(10)60503-x

9. Naidoo K, Singh J, Lalloo U. A critical analysis of ICU/HC beds in South Africa: 2008-2009. S Afr Med J 2013;103(10):751-753. https://doi.org/10.7196/samj.6415

10. Halpern NA, Pastores SM. Critical care medicine in the United States 2000 - 2005: An analysis of bed numbers, occupancy rates, payer mix, and costs. Crit Care Med 2010;38(1):65-71. https://doi. org/10.1097/ccm.0b013e3181b090do

11. Audit Commission. Critical to success. Portsmouth: Holbrooks Printers; 1999.

12. Prin $\mathrm{M}$, Wunsch $\mathrm{H}$. International comparisons of intensive care: Informing outcomes and improving standards. Curr Opin Crit Care 2012;18(6):700-706. https://doi.org/10.1097/mcc.0b013e32835914d5

13. Wunsch H, Angus DC, Harrison DA, Linde-Zwirble WT, Rowan KM. Comparison of medical admissions to intensive care units in the United States and United Kingdom. Am J Respir Crit Care admissions to intensive care units in the United States and United Kingdom.

14. Turner JS, Michell WL, Morgan CJ, Benatar SR. Limitation of life support: Frequency and practice in a London and a Cape Town intensive care unit. Intensive Care Med 1996;22:1020-1025. https:// doi.org/10.1007/bf01699222

15. Quill CM, Ratcliffe SJ, Harhay MO, Halpern SD. Variation in decisions to forgo life-sustaining therapies in US ICUs. Chest 2014;146(3):573-582. https://doi.org/10.1378/chest.13-2529

Accepted 18 December 2019 